\title{
Morphology and subsequent development in culture of bovine oocytes matured in vitro under various conditions of fertilization
}

\author{
C. R. Long ${ }^{1}$, P. Damiani ${ }^{1}$, C. Pinto-Correia ${ }^{1}$, R. A. MacLean ${ }^{2}$, \\ R. T. Duby ${ }^{1}$ and J. M. Robl ${ }^{1 *}$ \\ ${ }^{1}$ Department of Veterinary and Animal Science, University of Massachusetts, Amherst, MA 01003, USA; \\ and ${ }^{2}$ Bova-Max Incorporated, Hanford, CA 93230, USA
}

\begin{abstract}
The objective of these experiments was to evaluate factors affecting in vitro fertilization of bovine oocytes matured in vitro, and their subsequent development to blastocysts. In Expts 1 and 2, sperm concentration, spermatozoa and oocyte incubation time, motility enhancers and semen source were manipulated. Fluorescent microscopy of microtubules and chromatin was used to observe sperm penetration rate, sperm aster formation and chromatin decondensation. Oocyte penetration rates were affected by sperm concentration but not by spermatozoa and oocyte incubation time. The effect of sperm concentration was due primarily to changes in polyspermy and not monospermy. Motility enhancers had no effect on any parameter measured. In Expt 3, oocytes were matured for $17,22,28$ and $34 \mathrm{~h}$ before fertilization and evaluated for fertilization rates, morphology of cortical granules and exocytosis and blastocyst development. A domain free of cortical granules that was associated with the metaphase chromatin was not observed in mature bovine oocytes. As oocytes matured from 17 to $34 \mathrm{~h}$, the distribution of cortical granules progressed from clustered to diffuse. Although monospermic fertilization rates were similar and cortical granule exocytosis occurred in all groups, polyspermy increased with maturation time. Development to blastocysts increased from 17 to $22 \mathrm{~h}$ of maturation but decreased thereafter with increasing maturation time. These results suggest that polyspermy can be reduced by adjusting sperm concentration and spermatozoa and oocyte incubation time with little effect on monospermic fertilization. Increased polyspermy with increased maturation time was not due to a lack of cortical granule exocytosis.
\end{abstract}

\section{Introduction}

Recent experimentation in maturation, fertilization and culture of bovine oocytes in vitro has led to marked improvements in the production of embryos in vitro. These advances have allowed progress in the study of early fertilization events so that the efficiency of embryo production in vitro can be improved. Further manipulation of the bovine genome by cloning and gene transfer requires in vitro systems to produce large numbers of transferable, high-quality, diploid (with maternal and paternal chromosomes) embryos. The optimum system would give high penetration rates with minimal polyspermy or other abnormal fertilizations. However, abnormal ultrastructural features of in vitro matured (IVM) and in vitro fertilized (IVF) oocytes have been described by Hyttel et al. (1986, 1988, 1989), including delayed dispersal of cortical granules, incomplete cortical granule release and distribution, polyspermy and delayed pronuclear development. $X_{u}$ and

*Correspondence.

Revised manuscript received 18 April 1994.
Greve (1988) reported four types of abnormalities of IVF and IVM oocytes: polyspermy, polygyny, asynchronous pronuclear development and preactivation of cytokinesis. Although some of the developmental abnormalities have been corrected, polyspermy remains the most prevalent abnormality, as described by Saeki et al. (1991), Chian et al. (1992) and Pavlok et al. (1992). Previous reports have suggested that polyspermically fertilized bovine oocytes can develop to the morula and blastocyst stage, possibly in some cases as androgenotes (Iwasaki et al., 1989; Pinto-Correia et al., 1992; Long et al., 1993).

The objective of these experiments was to investigate factors affecting the penetration of spermatozoa into IVM bovine oocytes, and their further development to transferable embryos. Factors evaluated were sperm concentration, motility enhancers, duration of sperm incubation, source of spermatozoa and oocyte maturation time. Fluorescent microscopy of chromatin, microtubules and cortical granules was used to examine the interaction between spermatozoa and oocytes before and during the early stages after fertilization. The morphology of spermatozoa and oocytes after fertilization and 
possible reasons for polyspermy, as well as the relationship between fertilization rates and blastocyst development using IVM bovine oocytes, are presented.

\section{Materials and Methods}

\section{In vitro maturation}

In Expts 1 and 2, oocytes were obtained by aspirating follicles from ovaries obtained from an abattoir. Ovaries were placed in insulated containers with warm packs for transport to the laboratory. The ovaries arrived approximately $4 \mathrm{~h}$ after collection at an average temperature of $30^{\circ} \mathrm{C}$, and were washed in warm $0.9 \%(\mathrm{w} / \mathrm{v})$ saline solution. Follicles $1-10 \mathrm{~mm}$ in diameter were aspirated using an 18-gauge needle attached to a collection tube, with a pressure release valve in line to a vacuum pump set to $100 \mathrm{~mm} \mathrm{Hg}$. The proportion of follicles $<2 \mathrm{~mm}$ in diameter was small and all recovered oocytes were selected for an intact cumulus and evenly pigmented cytoplasm, thus reducing the negative effects of using small follicles (Pavlok et al., 1992).

The selected cumulus-enclosed oocytes were washed twice in Tyrode-Hepes medium (TL Hepes; Bavister et al., 1983) supplemented with $3 \mathrm{mg} \mathrm{BSA} \mathrm{ml}{ }^{-1}, 1.0 \%$ penicillin and $1.0 \%$ streptomycin $\left(100 \mathrm{U} \mathrm{ml}^{-1}\right.$ and $100 \mathrm{mg} \mathrm{ml}^{-1}$, respectively; Gibco Laboratories, Grand Island, NY) and transferred to four-well tissue culture plates (Nunclon, VWR Scientific, Bridgeport, NJ) containing $250 \mu \mathrm{l}$ maturation medium per well. Maturation medium consisted of TCM 199 with Earle's salts (Gibco Laboratories) supplemented with $10 \%(\mathrm{v} / \mathrm{v})$ heat-treated fetal bovine serum (lot number 11112135; Hyclone Labs, Logan, UT), I ng oestradiol $\mathrm{ml}^{-1}$ (Sigma Chemical Co., St Louis, MO), $0.5 \mu \mathrm{g}$ ovine FSH ml ${ }^{-1}$ [National Institute of Diabetes and Digestive and Kidney Diseases (NIDDKD), Baltimore, MDl and $5.0 \mu \mathrm{g}$ bovine $\mathrm{LH}-5 \mathrm{ml}^{-1}$ (NIDDKD). Fetal bovine serum and the media used in all the experiments were from the same lot, except the maturation medium used in Expt 3. Oocytes were incubated under a humidified atmosphere of $5 \% \mathrm{CO}_{2}$ in air at $38.5^{\circ} \mathrm{C}$ for $22 \mathrm{~h}$, and routinely $>90 \%$ of oocytes that were produced had metaphase II plates.

Oocytes for Expt 3 were acquired from BovaMax Incorporated, Hanford, CA. Oocytes were obtained by harvesting ovaries from an abattoir and aspirating follicles (approximately $3 \mathrm{~h}$ after collection), as described above. Cumulusenclosed oocytes were washed twice in TL Hepes, and approximately 50 oocytes were transferred to each $1.8 \mathrm{ml}$ tissue culture tube containing $500 \mu \mathrm{l}$ of pre-equilibrated maturation medium covered to capacity with paraffin oil (Dow Corning 500). BovaMax maturation medium consisted of TCM 199 with Earle's salts supplemented with $10 \%(\mathrm{v} / \mathrm{v})$ heat-treated fetal bovine serum (Hyclone Labs) $0.01 \mathrm{U}$ bovine $\mathrm{LH} \mathrm{ml}^{-1}$ (Nobl, Sioux Center, IA), $0.01 \mathrm{U}$ bovine FSH ml ${ }^{-1}$ $(\mathrm{Nobl})$ and $1.0 \%(\mathrm{w} / \mathrm{v})$ penicillin and $1.0 \%(\mathrm{w} / \mathrm{v})$ streptomycin The tightly sealed tubes were labelled with the starting time of maturation and placed in a battery-powered incubation shipper (Minitube of America, Incorporated, Madison, WI) for transport via overnight express service to our laboratory. The portable incubator temperature was recorded and averaged $38.5^{\circ} \mathrm{C}$ at the end of the $15-17 \mathrm{~h}$ shipment. The tubes were moved to the maturation incubator until the time of insemination, when the cumulus-enclosed oocytes were washed three times in warm TL Hepes and transferred to fertilization medium. Maturation in this system was effective in producing mature oocytes, since $<5 \%$ oocytes had not progressed to metaphase II after $17-22 \mathrm{~h}$ in maturation medium followed by $8 \mathrm{~h}$ in fertilization medium without spermatozoa.

\section{In vitro fertilization and culture}

The fertilization medium consisted of CR1aa (Rosenkrans and First, 1991) supplemented with $6 \mathrm{mg}$ fatty-acid-free BSA $\mathrm{ml}^{-1}, 5.0 \mu \mathrm{g}$ sodium heparin $\mathrm{ml}^{-1}$ (Sigma Chemical Co.) to induce sperm capacitation (Parrish et al., 1988) and $20 \mu \mathrm{mol}$ penicillamine $1^{-1}, 10 \mu \mathrm{mol}$ hypotaurine $1^{-1}$ and $1 \mu \mathrm{mol}$ adrenaline $1^{-1}$ (PHE) to stimulate sperm motility unless otherwise specified. Oocytes were fertilized in groups of $30-50$ in four-well tissue culture plates in a final volume of $500 \mu \mathrm{l}$ fertilization medium incubated under a humidified atmosphere of $5 \% \mathrm{CO}_{2}$ in air at $38.5^{\circ} \mathrm{C}$. Semen that had been collected from five sires, pooled, diluted in a milk-based extender and cryopreserved was donated by Eastern AI Cooperative, Incorporated (Ithaca, NY). This semen was used in Expts 1 and 3, and as a control in Expt 2. Oocytes were inseminated using frozen-thawed spermatozoa that had been separated from the extender using a Percoll gradient $(45 \% / 90 \%)$; the resulting sperm pellet was resuspended in the fertilization medium to desired concentrations. After incubation with spermatozoa, the oocytes were placed in a $15 \mathrm{ml}$ conical tube with $0.5-0.75 \mathrm{ml}$ warm TL Hepes and vortexed using a Genie Vortex 2 (Fisher Scientific, Pittsburgh, PA) on the highest speed for $2 \mathrm{~min}$ and $15 \mathrm{~s}$ to remove the cumulus cells.

Inseminated oocytes were cultured in $50 \mu \mathrm{l}$ drops of CRTaa supplemented with $10 \%$ heat-treated fetal bovine serum (CR2) under paraffin oil. Inseminated oocytes $(n=10-20)$ were cultured in each drop of CR2, without changing or adding CR2, for a total of 8 days (day 0 : day of insemination). These conditions allowed a cumulus cell monolayer to be established from those remaining attached to the zona pellucida after vortexing. Development was calculated on day 8 as the number of expanding or hatching blastocysts, with no or a few extruded or dead cells (grade 1 or 2 , respectively), divided by the total number of oocytes cultured.

\section{Immunolocalization of chromatin and microtubules}

In Expts $I$ and 3, chromatin and microtubules were detected by immunofluorescence of whole mounts of zygotes, as described by Long et al. (1993). Zygotes were transferred to a microtubule stabilization buffer (Schatten et al., 1985) containing $3 \%$ Triton $\mathrm{X}-100(\mathrm{v} / \mathrm{v})$ for $6 \mathrm{~h}$ at $38^{\circ} \mathrm{C}$, which was repeatedly changed, followed by fixation in $2.0 \%(\mathrm{v} / \mathrm{v})$ paraformaldehyde in PBS for $30 \mathrm{~min}$ at $38^{\circ} \mathrm{C}$. After washing in PBS and incubating for $10 \mathrm{~min}$ in $0.1 \mathrm{~mol}$ glycine $1^{-1}$, the zygotes were incubated overnight at $4^{\circ} \mathrm{C}$ with anti- $\alpha$-tubulin monoclonal antibody (Sigma Chemical $\mathrm{Co}$.) diluted 1:100 in PBS containing $0.1 \%(\mathrm{v} / \mathrm{v})$ Tween $20(\mathrm{PBS}-\mathrm{T})$. Zygotes were washed for a minimum of $6 \mathrm{~h}$ with several changes of PBS-T 
and incubated overnight in fluorescein isothiocyanateconjugated, affinity-purified goat anti-mouse immunoglobulin G (IgG) (FITC; Sigma Chemical Co.) diluted 1:10 in PBS-T at $4^{\circ} \mathrm{C}$. After incubation, zygotes were washed for a minimum of $6 \mathrm{~h}$ with repeated changes of PBS-T and mounted on glass slides in glycerol containing $0.5 \mu \mathrm{g}$ Hoechst $33342 \mathrm{ml}^{-1}$ (Sigma Chemical Co.). Coverslips were sealed to slides with nail polish and stored in the dark at $4^{\circ} \mathrm{C}$ 1-7 days before evaluation. Control zygotes were incubated with 1:100 affinity-purified mouse IgG (Sigma Chemical Co.) instead of $\alpha$-tubulin antibody, and always gave negative results.

A direct method of visualizing the chromatin without staining the microtubules was used in Expt 2, since only the sperm penetration rate and developmental capacity were evaluated. After cumulus removal, zygotes were transferred to $3.7 \%$ $(\mathrm{v} / \mathrm{v})$ paraformaldehyde containing $10.0 \%(\mathrm{v} / \mathrm{v})$ Triton $\mathrm{X}-100$ for $20 \mathrm{~min}$ at $38^{\circ} \mathrm{C}$, followed by extensive washing in PBS containing $0.3 \%(\mathrm{w} / \mathrm{v}) \mathrm{BSA}$. Oocytes were mounted in glycerol with Hoechst as above. Cell counts of blastocysts 8 days after insemination produced in Expt 2 were performed by mounting grade 1 and 2 embryos directly to glycerol with Hoechst on glass slides, and counting nuclei.

\section{Cortical granule localization and staining}

The staining procedure was modified from previously published reports using hamster oocytes (Cherr et al., 1988). After vortexing to remove the cumulus cells, oocytes and presumptive zygotes were treated with $0.4 \%(\mathrm{w} / \mathrm{v})$ pronase E (Sigma Chemical Co.) to remove the zona pellucida. The nude cells were washed three times in TL Hepes and transferred to $3.0 \%$ $(\mathrm{v} / \mathrm{v})$ paraformaldehyde in PBS for $\mathrm{I} \mathrm{h}$ at room temperature. Fixed cells were washed in blocking solution (PBS containing $1.0 \%(\mathrm{w} / \mathrm{v}) \mathrm{BSA}, 0.1 \mathrm{~mol}$ glycine $1^{-1}$ and $0.05 \%(\mathrm{v} / \mathrm{v})$ Triton $X-100$ ) for $1 \mathrm{~h}$ and $40 \mathrm{~min}$ with five changes of solution. Cells were transferred to fluorescein isothiocyanate conjugated with Lens culinaris agglutinin (10 $\mu \mathrm{g} \mathrm{ml}^{-1}$; Sigma Chemical Co.) in blocking solution for $30 \mathrm{~min}$ in the dark at room temperature. Labelled cells were washed in blocking solution as above, followed by a similar wash in PBS containing $1 \mathrm{mg}$ polyvinylpyrrolidone $\mathrm{ml}^{-1}$, and mounted in glycerol with Hoechst. This procedure was first tested using mature mouse oocytes and resulted in cortical granule patterns as described by Ducibella et al. (1988).

\section{Microscopy and photography}

All oocytes or zygotes stained for chromatin, microtubules or cortical granules were visualized at $\times 1000$ using a Zeiss Axioskop microscope equipped with epifluorescence. Hoechst and FITC signals were detected using 365/400 and 450/520 nm excitation/barrier filter combinations, respectively. A video camera attached to the microscope was used to capture images that were produced using the Image I AT software (Universal Imaging, Media, PA). Images were stored in $44 \mathrm{Mb}$ disk cartridges, and photomicrographs were prepared with a video printer (model UP5000; Sony, Park Ridge, NJ). Blastocyst nuclei were counted at $\times 200$ using a Nikon Diaphot microscope equipped with epifluorescence and Hoechst filter combinations as above.

\section{Experimental design}

Experiment 1. The objective of Expt 1 was to investigate the effects of sperm concentration, spermatozoa and oocyte incubation time and the presence or absence of PHE on sperm penetration rates and subsequent development in vitro in a $3 \times 2 \times 2$ factorial design. All oocytes were inseminated after $22 \mathrm{~h}$ of maturation using the pooled spermatozoa as described above at concentrations of $0.5,0.25$ and $0.125 \times 10^{6}$ spermatozoa $\mathrm{ml}^{-1}$ in the presence or absence of PHE. Oocytes were removed from the fertilization medium 8 or $18 \mathrm{~h}$ after insemination. Oocytes vortexed $8 \mathrm{~h}$ after insemination were cultured in CR2; a proportion of these oocytes $(n=253)$ were removed and fixed $10 \mathrm{~h}$ later to evaluate fertilization rates, leaving 347 oocytes in CR2. Oocytes vortexed $18 \mathrm{~h}$ after insemination were divided into groups to be fixed immediately $(n=295)$ or cultured in CR2 $(n=380)$. Data were collected over three replicates $(n=384,449,442$ for replicates 1,2 and 3 , respectively) and included degree of sperm penetration, abnormal stages of development and the number of oocytes developing to blastocysts.

Experiment 2. The effect of sire and sperm concentration on the fertilization of IVM bovine oocytes and their subsequent development was evaluated in Expt 2 in a $5 \times 3$ factorial design. Semen from four sires of known non-return rates (A: 38\%; B: 64\%; C: 68\%; D: 73\%) and the pooled control semen (described above) were compared for fertilization rates and blastocyst development. Spermatozoa from each source were prepared at concentrations of $1.0,0.5$ and $0.25 \times 10^{6}$ spermatozoa $\mathrm{ml}^{-1}$ as described, and combined with oocytes that had matured for $22 \mathrm{~h}$. Oocytes and spermatozoa were co-incubated for $8 \mathrm{~h}$ in fertilization medium containing PHE and $5 \mu \mathrm{g}$ sodium heparin $\mathrm{ml}^{-1}$. Cumulus cells were removed by vortexing and a proportion of zygotes from each treatment group was fixed for chromatin evaluation $(n=890)$. The remainder $(n=1206)$ was cultured in drops of CR2 to evaluate development to the blastocyst stage. The experiment was replicated six times $(n=291-510$ oocytes per replicate).

Experiment 3. The effect of oocyte age on the block to polyspermy and on nuclear and cytoskeletal morphology during the first cell cycle and subsequent development to blastocysts were evaluated in nine replicates of Expt 3 . Oocytes were maintained in maturation medium for $17,22,28$ or $34 \mathrm{~h}$ and then moved to fertilization medium containing PHE and $0.5 \times 10^{6}$ spermatozoa $\mathrm{ml}^{-1}$. This combination of spermatozoa and PHE was used because it had induced high rates of polyspermy in previous experiments and therefore challenged the oocytes block to polyspermy at each period. Presumptive zygotes were vortexed $8 \mathrm{~h}$ after insemination and fixed immediately, or after $10 \mathrm{~h}$ of culture in CR2, to evaluate sperm penetration, sperm aster formation and pronuclear development ( $n=657$; range: $69-109$ per replicate). In addition, 1473 (range: 111-202 per replicate) presumptive zygotes were cultured for 8 days in CR2 to compare developmental capability between treatments. 
Table 1. Fertilization rates and blastocyst development of bovine oocytes inseminated with different concentrations of spermatozoa and for different incubation times in vitro

\begin{tabular}{|c|c|c|c|c|c|c|}
\hline \multirow[b]{2}{*}{ Parameter } & \multicolumn{4}{|c|}{ Fixed } & \multicolumn{2}{|c|}{ Cultured } \\
\hline & $n$ & $2 \mathrm{PN}(\%)^{\mathrm{c}}$ & PPN $(\%)^{d}$ & PENT $(\%)^{\mathrm{c}}$ & $n$ & Blastocysts $(\%)^{f}$ \\
\hline \multicolumn{7}{|c|}{ Sperm concentration $\left(\times 10^{6} \mathrm{ml}^{-1}\right)$} \\
\hline 0.5 & 188 & $49.4^{\mathrm{a}}$ & $36.4^{a}$ & $85.8^{\mathrm{a}}$ & 262 & $28.4^{\mathrm{a}}$ \\
\hline 0.25 & 183 & $55.9^{a}$ & $15.9^{b}$ & $71.8^{b}$ & 211 & $22.9^{\mathrm{ab}}$ \\
\hline 0.125 & 177 & $\begin{array}{l}50.5^{\mathrm{a}} \\
(4.72)\end{array}$ & $\begin{array}{l}10.2^{b} \\
(4.36)\end{array}$ & $\begin{array}{l}60.7^{b} \\
(4.57)\end{array}$ & 254 & $\begin{array}{l}15.3^{\mathrm{b}} \\
(4.35)\end{array}$ \\
\hline \multicolumn{7}{|c|}{ Sperm incubation time $(\mathrm{h})$} \\
\hline 18 & 295 & $48.3^{\mathrm{a}}$ & $25.9^{\mathrm{a}}$ & $74.2^{a}$ & 380 & $23.4^{\mathrm{a}}$ \\
\hline 8 & 253 & $\begin{array}{l}55.5^{\mathrm{a}} \\
(3.85)\end{array}$ & $\begin{array}{l}15.7^{\mathrm{b}} \\
(3.56)\end{array}$ & $\begin{array}{l}71.2^{\prime \prime} \\
(3.73)\end{array}$ & 347 & $\begin{array}{l}21.1^{\mathrm{a}} \\
(3.55)\end{array}$ \\
\hline
\end{tabular}

\footnotetext{
a.b Values with different superscripts within a column are significantly different $(P<0.05$, analysed by SAS least squares means; SEM is given in parentheses.

'Percentage of fixed oocytes penetrated by only one spermatozoon.

Percentage of fixed oocytes penetrated by two or more spermatozoa.

ePercentage of fixed oocytes penetrated by at least one spermatozoon.

fPercentage of inseminated oocytes developing to grade 1 and 2 blastocysts on day 8 .
}

In the last three of nine replicates, a portion of unfertilized oocytes from each age group was fixed to determine cortical granule patterns ( $n=35 \pm 5$ per replicate). Cortical granule patterns and sperm penetration rates were determined in 201 inseminated oocytes over the three replicates. After maturation for the appropriate time, oocytes were moved to fertilization medium with or without spermatozoa for $8 \mathrm{~h}$, vortexed and fixed immediately.

\section{Statistical analyses}

Data for all experiments were analysed by the General Linear Models (GLM) and Least Squares Means (LSM) procedures (SAS Institute Inc., Cary, NC) to test for significance of main effects and differences between treatments. Arcsin transformations were used when testing significance, although the nontransformed LSM results are presented in tables and text. In Expt 1, PHE, sperm concentration and sperm incubation time were main effects and all possible interactions were tested. The main effects of sire and sperm concentration as well as their interaction with oocytes were tested in Expt 2. In Expt 3 the effect of oocyte age on fertilization rate, cortical granule morphology and exocytosis and blastocyst development was tested. In all experiments a replicate was a percentage calculated from the total number of oocytes in a treatment from each day.

\section{Results}

\section{Experiment 1: sperm concentration, sperm incubation time and PHE}

Addition of PHE to the fertilization medium did not increase the number of oocytes penetrated by one or more spermatozoa ( $75.9 \%$ versus $69.6 \%$, with and without PHE, respectively), monospermic $(52.7 \%$ versus $51.1 \%$, with and without $\mathrm{PHE}$, respectively) and polyspermic ( $23.1 \%$ versus $18.5 \%$, with and without PHE, respectively) penetration rates or blastocyst development $(23.8 \%$ versus $20.7 \%$, with and without PHE, respectively). There was no interaction between the effects of PHE, sperm concentration or time of sperm removal; therefore, data were subsequently analysed only for the main effects of sperm concentration and the time of sperm removal.

Polyspermic and total penetration rates were greater for $0.5 \times 10^{6}$ spermatozoa $\mathrm{ml}^{-1}$ than for other sperm concentrations $(P<0.05)$. This resulted in an increased development to the blastocyst stage compared with a concentration of $0.125 \times 10^{6}$ spermatozoa $\mathrm{ml}^{-1}$, although monospermic fertilization rates were not different among sperm concentrations $(P>0.3)$ (Table 1).

Incubation of oocytes and spermatozoa for $18 \mathrm{~h}$ resulted in a higher rate of polyspermy $(P<0.05)$ but did not affect monospermic fertilization or development to blastocysts (Table 1). There was an increase in the number of zygotes fixed during anaphase or telophase II within the $18 \mathrm{~h}$ treatment $(7.0 \%$ versus $0.0 \%$ for 18 and $8 \mathrm{~h}$, respectively; $P<0.01$ ). Chromatin anomalies also increased as sperm incubation time increased $(8.2 \%$ versus $3.8 \%$ for 18 and $8 \mathrm{~h}$, respectively; $P<0.05$ ). These anomalies included double and split spindles, extrusion of the maternal chromatin and others that have been described by Long et al. (1993).

\section{Experiment 2: sperm source}

The effect of sire on fertilization rates and blastocyst development is shown in Table 2. Of the three sperm concentrations used per sire, $0.25 \times 10^{6}$ spermatozoa ml $\mathrm{ml}^{-1}$ yielded the lowest oocyte penetration rate and no significant sire by concentration interactions were observed (data not shown). Therefore, the data for sperm concentration were 
Table 2. Fertilization rates and blastocyst development of bovine oocytes inseminated with different semen sources in vitro

\begin{tabular}{|c|c|c|c|c|c|c|c|}
\hline \multirow[b]{2}{*}{ Semen source } & \multicolumn{4}{|c|}{ Fixed } & \multicolumn{3}{|c|}{ Cultured } \\
\hline & $n$ & $2 \mathrm{PN}(\%)^{\mathrm{d}}$ & $\operatorname{PPN}(\%)^{\mathrm{e}}$ & PENT $(\%)^{f}$ & $n$ & Blastocysts $(\%)^{g}$ & Number of cells per embryo \\
\hline A & 139 & $\begin{array}{c}5.4^{\mathrm{a}} \\
(4.02)\end{array}$ & $\begin{array}{c}0.0^{\mathrm{a}} \\
(3.38)\end{array}$ & $\begin{array}{c}5.4^{\mathrm{a}} \\
(8.73)\end{array}$ & 194 & $\begin{array}{c}0.5^{\mathrm{a}} \\
(3.80)\end{array}$ & $100.0^{a}$ \\
\hline B & 190 & $\begin{array}{l}60.6^{\mathrm{b}} \\
(4.02)\end{array}$ & $\begin{array}{l}11.5^{\mathrm{b}} \\
(3.38)\end{array}$ & $\begin{array}{l}72.1^{\mathrm{b}} \\
(8.73)\end{array}$ & 205 & $\begin{array}{l}22.9^{b} \\
(3.80)\end{array}$ & $119.3^{\mathrm{a}}$ \\
\hline C & 190 & $\begin{array}{l}67.8^{\mathrm{b}} \\
(3.67)\end{array}$ & $\begin{array}{l}23.9^{c} \\
(3.08)\end{array}$ & $\begin{array}{l}91.7^{c} \\
(7.97)\end{array}$ & 267 & $\begin{array}{l}30.2^{\mathrm{b}} \\
(3.47)\end{array}$ & $120.1^{\mathrm{a}}$ \\
\hline $\mathrm{D}$ & 185 & $\begin{array}{l}65.7^{\mathrm{b}} \\
(3.67)\end{array}$ & $\begin{array}{l}17.5^{\mathrm{bc}} \\
(3.08)\end{array}$ & $\begin{array}{l}83.2^{\mathrm{c}} \\
(7.97)\end{array}$ & 267 & $\begin{array}{l}27.3^{b} \\
(3.47)\end{array}$ & $109.5^{\mathrm{a}}$ \\
\hline Pooled & 186 & $\begin{array}{l}73.6^{\mathrm{b}} \\
(5.19)\end{array}$ & $\begin{array}{l}17.1^{\mathrm{bc}} \\
(4.36)\end{array}$ & $\begin{array}{c}90.7^{\mathrm{c}} \\
(11.27)\end{array}$ & 273 & $\begin{array}{l}33.8^{\mathrm{b}} \\
(4.91)\end{array}$ & $105.3^{2}$ \\
\hline
\end{tabular}

a,b,c Values with different superscripts within a column are significantly different $(P<0.05$, analysed by SAS least squares means; SEM is given in parentheses.

${ }^{d}$ Percentage of fixed oocytes penetrated by only one spermatozoon.

ePercentage of fixed oocytes penetrated by two or more spermatozoa.

fPercentage of fixed oocytes penetrated by at least one spermatozoon.

${ }^{8}$ Percentage of inseminated oocytes developing to grade 1 and 2 blastocysts on day 8 .

Table 3. Fertilization rates of blastocyst development of bovine oocytes following different maturation times

\begin{tabular}{|c|c|c|c|c|c|c|}
\hline \multirow[b]{2}{*}{ Maturation time (h) } & \multicolumn{4}{|c|}{ Fixed } & \multicolumn{2}{|c|}{ Cultured } \\
\hline & $n$ & $2 \mathrm{PN}(\%)^{\mathrm{d}}$ & PPN $(\%)^{e}$ & PENT $(\%)^{f}$ & $n$ & Blastocysts $(\%)^{\mathrm{g}}$ \\
\hline 17 & 150 & $\begin{array}{r}45.1^{\mathrm{a}} \\
(4.4)\end{array}$ & $\begin{array}{r}37.0^{a} \\
(3.8)\end{array}$ & $\begin{array}{l}82.1^{\mathrm{a}} \\
(2.6)\end{array}$ & 366 & $\begin{array}{r}21.6^{a} \\
(3.1)\end{array}$ \\
\hline 22 & 157 & $\begin{array}{r}47.6^{\mathrm{a}} \\
(4.4)\end{array}$ & $\begin{array}{c}42.9^{\mathrm{abb}} \\
(3.8)\end{array}$ & $\begin{array}{c}90.5^{\mathrm{b}} \\
(2.6)\end{array}$ & 353 & $\begin{array}{c}38.3^{\mathrm{b}} \\
(3.1)\end{array}$ \\
\hline 28 & 192 & $\begin{array}{r}47.7^{\mathrm{a}} \\
(5.0)\end{array}$ & $\begin{array}{r}51.0^{b} \\
(4.4)\end{array}$ & $\begin{array}{r}98.7^{\mathrm{c}} \\
(2.9)\end{array}$ & 399 & $\begin{array}{c}30.1^{\mathrm{ab}} \\
(3.5)\end{array}$ \\
\hline 34 & 158 & $\begin{array}{r}39.3^{\mathrm{a}} \\
(4.7)\end{array}$ & $\begin{array}{r}56.2^{\mathrm{b}} \\
(4.1)\end{array}$ & $\begin{array}{l}95.5^{\mathrm{bc}} \\
(2.8)\end{array}$ & 355 & $\begin{array}{l}11.2^{\mathrm{c}} \\
(3.3)\end{array}$ \\
\hline
\end{tabular}

\footnotetext{
${ }^{\text {s.b. }}$ Values with different superscripts within a column are significantly different $(P<0.05$, analysed by sAs least squares means; SEM is given in parentheses.

${ }^{\mathrm{P}}$ Percentage of fixed oocytes penetrated by only one spermatozoon.

ePercentage of fixed oocytes penetrated by two or more spermatozoa.

'Percentage of fixed oocytes penetrated by at least one spermatozoon.

sPercentage of inseminated oocytes developing to grade 1 and 2 blastocysts on day 8 .
}

pooled within sire. Sire A had a lower oocyte penetration rate and led to a lower embryo development rate than did other semen sources tested $(P<0.001)$. Although the semen from sire $B$ tended to give poorer results $(P=0.07)$, no significant differences were observed between individual sires $B, C$ and $D$ and the pooled control semen with respect to monospermic fertilization and development to blastocysts. Sire B displayed lower polyspermy than sire $C(P<0.01)$. Polyspermic fertilization rates were not different among sires $C, D$ and pooled control. The oocyte penetration rate of semen from sire $\mathrm{B}$ was lower than that of sires $C, D$ and pooled control $(P<0.05)$, mainly due to decreased polyspermy. In addition, the number of blastomeres per blastocyst on day 8 of culture was not different among sires and pooled control semen.

\section{Experiment 3: oocyte ageing}

Increasing oocyte maturation time from 17 to $34 \mathrm{~h}$ had no effect on monospermic fertilization of bovine oocytes (Table 3). The number of oocytes penetrated by at least one spermatozoon was lower for oocytes matured for $17 \mathrm{~h}(P<0.05)$, due to a decrease in polyspermic fertilization. Blastocyst development was lowest for oocytes inseminated after $34 \mathrm{~h}$ of maturation $(P<0.05)$; a maturation time of $17 \mathrm{~h}$ resulted in lower development rates than periods of 22 or $28 \mathrm{~h}(P<0.05)$, while development tended to be highest in oocytes inseminated after $22 \mathrm{~h}$ of maturation $(P<0.10)$.

Evaluation of chromatin decondensation and sperm aster formation was made from zygotes fixed $8 \mathrm{~h}$ after insemination. 

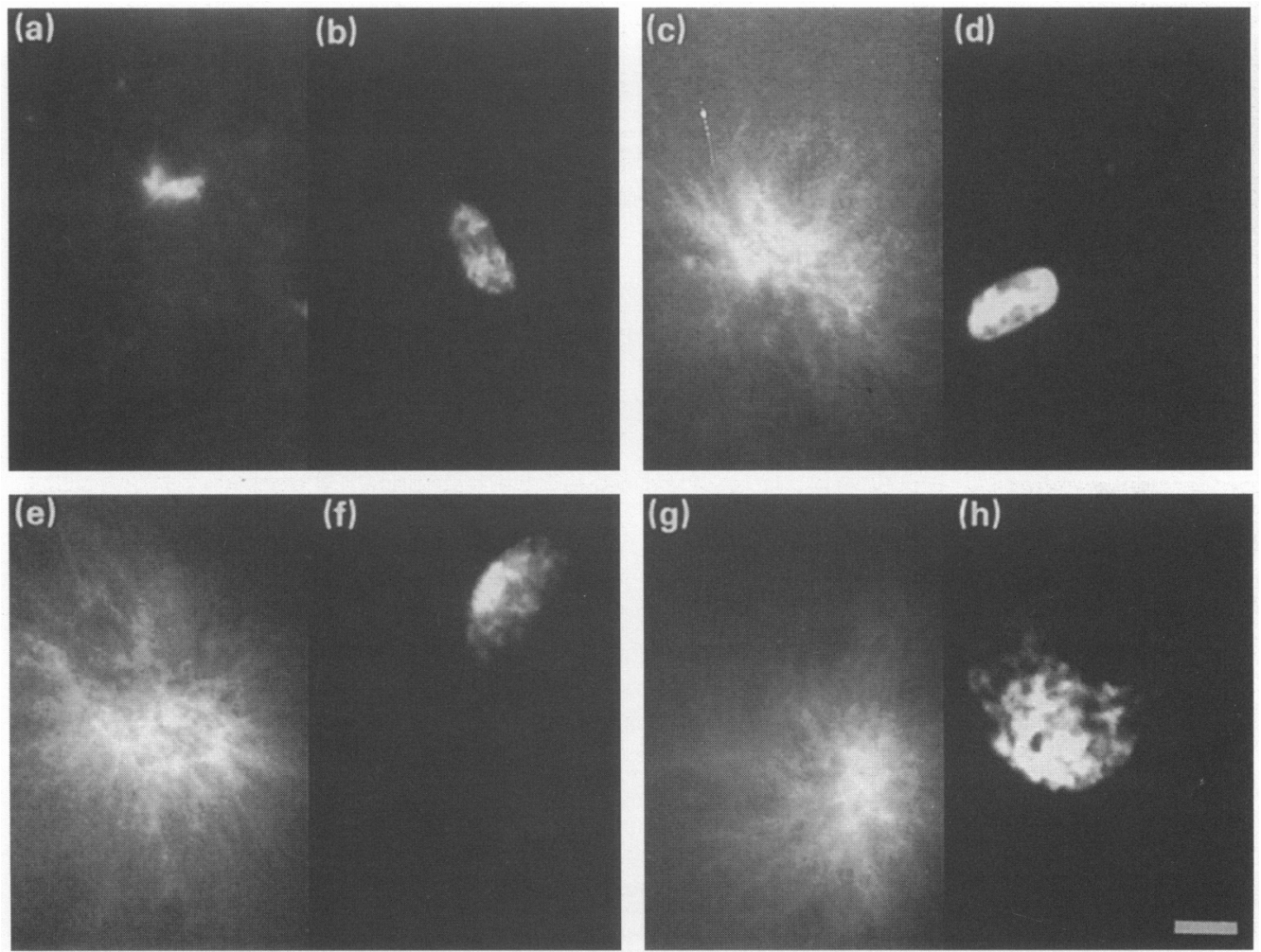

Fig. 1. Sperm-derived microtubules and chromatin decondensation patterns of bovine oocytes matured in vitro. Oocytes were fixed following $8 \mathrm{~h}$ of incubation with spermatozoa after they had matured for $25(\mathrm{a}, \mathrm{b}), 30$ (c, d), $36(\mathrm{e}, \mathrm{f})$ or $42(\mathrm{~g}, \mathrm{~h}) \mathrm{h}$. The figure shows (a) a small microtubule aster radiating from the tip of the sperm tail and (b) a slightly decondensed sperm head in an oocyte that had been fertilized and fixed after maturation for $25 \mathrm{~h}$. A large microtubule aster ( $c, e, g)$ and a decondensed sperm head $(d, f)$ or pronucleus $(h)$ can be observed in oocytes that had been fertilized and fixed after 30,36 and $42 \mathrm{~h}$ of maturation. Scale bar represents $10 \mu \mathrm{m}$.

Figure la illustrates the small and irregularly shaped aster extending from the midpiece of the spermatozoon from an oocyte inseminated after $17 \mathrm{~h}$ of maturation. The sperm head is still compact having undergone little decondensation (Fig. 1b). This is in contrast to sperm aster development and sperm head decondensation observed in oocytes fertilized at later times. Oocytes inseminated after 22 and $28 \mathrm{~h}$ of maturation (Fig. Ic, e) developed large sperm asters, accompanied by moderately decondensing sperm heads (Fig. 1d, ff. Insemination after $34 \mathrm{~h}$ of maturation resulted in large expanded microtubule asters and greatly decondensed sperm heads (Fig. Ig, h). Microtubule and chromatin decondensation patterns of oocytes matured for $34 \mathrm{~h}$ were similar to those observed in oocytes matured for $22 \mathrm{~h}$ and fixed $18 \mathrm{~h}$ after insemination.

Cortical granule distribution was analysed in fertilized and unfertilized oocytes within each maturation period and is illustrated in Fig. 2. After incubation for $8 \mathrm{~h}$ in fertilization medium without spermatozoa, the cortical granules of oocytes that had matured for $17 \mathrm{~h}$ were predominantly clustered (Fig. 2a). As maturation time increased, cortical granules dispersed. In oocytes that had matured for $22 \mathrm{~h}$, cortical granules were often still in small clusters, but after 28 or $34 \mathrm{~h}$ of maturation they were mostly dispersed (Fig. 2c, e, g). No domain that was free of cortical granules was ever observed. The chromatin was generaily in metaphase II as expected. Cortical granule exocytosis occurred in fertilized oocytes at all maturation times (Fig. 2b, d, f, h). The completeness of cortical granule exocytosis, however, increased with increasing maturation time, even though the rate of polyspermy increases as the maturation time increases.

\section{Discussion}

Maturation in Expts 1 and 2 was performed in different media and different incubation conditions than in Expt 3. Although no direct comparisons were made between these groups, it is noteworthy that oocytes matured during transport to our laboratory fertilized and developed to blastocysts at comparable rates to those matured in our laboratory. These data suggest that viable oocytes can be obtained from oocytes collected at distant locations and transported during the maturation period.

Sperm penetration occurs over an extended period, as oocytes cultured with spermatozoa for $18 \mathrm{~h}$ often had condensed sperm heads as well as anaphase or telophase chromatin arrangements at the time of fixing. In addition, sperm heads were observed in oocytes containing pronuclear structures, indicating penetration of previously fertilized oocytes. Decreasing the incubation time with spermatozoa decreases the rate of polyspermy while still resulting in similar monospermic penetration and development to the blastocyst stage. These 

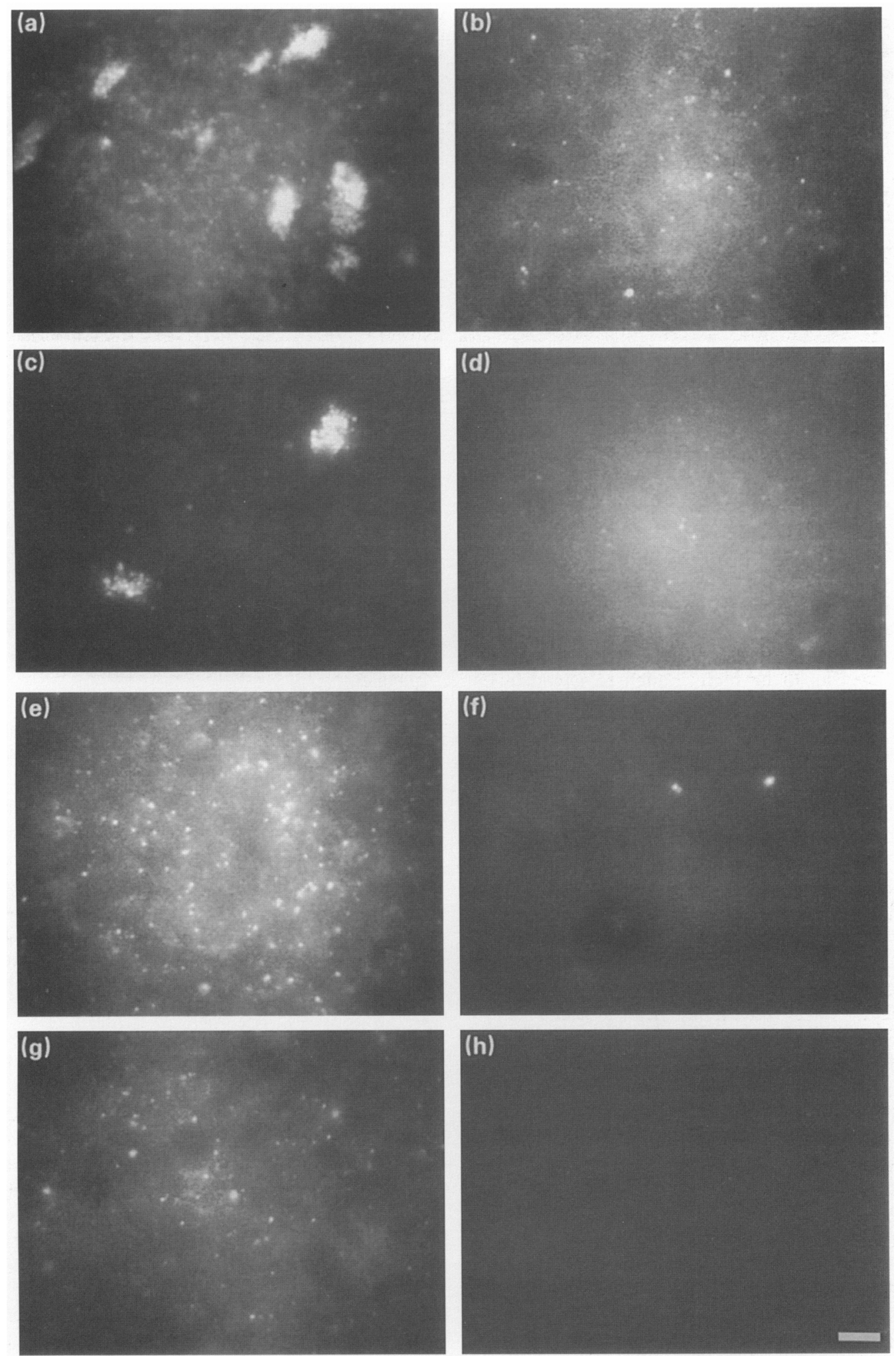

Fig. 2. Cortical granule patterns of bovine oocytes matured in iitro before and after sperm penetration. Oocytes were fixed following $8 \mathrm{~h}$ of incubation in fertilization medium with or without spermatozoa after the oocytes had matured for $25 \mathrm{~h}(\mathrm{a}, \mathrm{b}), 30 \mathrm{~h} \mathrm{c}, \mathrm{d}), 3 \mathrm{ch}(\mathrm{c}, \mathrm{f})$ and $+2 \mathrm{~h}(\mathrm{~g}, \mathrm{~h})$. The figure shows clustered cortical granules of oocytes that had matured for $25 \mathrm{~h}$ a before and blater fertilization. Iening slight traces of stainable material. (c) Oocytes that had matured for 30 h contained small clusters of cortical granules, but no staining was visible following fertilization (d). (c, s) Oocytes that had matured for $30 \mathrm{~h}$ and $42 \mathrm{~h}$ displayed well-distributed cortical granule staining but no staining was visible after fertilization $(f, h)$. Scale bar represents $10 \mu m$. 
data support previous reports by Saeki et al. (1991) and suggest that decreasing the spermatozoa and egg incubation time reduces undesirable polyspermic or anomalous fertilizations but does not result in significant improvements in monospermic fertilization or development to blastocysts.

Increasing the sperm concentration increased the incidence of polyspermy without affecting the number of monospermic penetrations. However, the rate of development to blastocysts was increased, suggesting that some blastocysts may be polyploid, androgenetic or able to eliminate accessory spermatozoa, as described by Long et al. (1993). Alternatively, the presence of numerous accessory spermatozoa during fertilization could produce a stimulatory effect on development in vivo, as described by DeJarnette et al. (1992) and Nadir et al. (1993). Lowering the sperm concentration to decrease the rate of polyspermy did not result in increased monospermic fertilizations and subsequent development to a transferable stage. However, if polyspermic zygotes develop to the blastocyst stage, this would have a deleterious effect on pregnancy rates after transfer to recipients.

The addition of PHE had no effect on any of the observed parameters in Expt 1, which is contrary to effects reported by Susko-Parrish et al. (1990). The positive influence of motility enhancers might be observed in fertilization systems, in which penetration rates are low. Over the three experiments described, sperm penetration of control oocytes was greater than $85 \%$ and appears to be high enough to eliminate the positive effects of PHE.

The source of semen affected the fertilization rate and subsequent development to blastocysts in these experiments. Although a limited number of sires were used, these results support previous observations by Parrish et al. (1986), Ohgoda et al. (1988) and others suggesting that the in vitro fertilization rate of bovine sires is highly variable.

As previously reported, in vitro maturation time of oocytes affects fertilization rates and development to blastocysts in vitro (Chian et al., 1992; Dominko and First, 1992; van der Westerlaken et al., 1992), as well as cortical granule distribution (Sathananthan and Trounson, 1982; Cran and Esper, 1990). The aim of the experiments described here was to focus on the mechanisms of fertilization or developmental failure in oocytes that had matured for short or long periods.

Reports by $\mathrm{Xu}$ and Greve (1988) indicate that without prior capacitation procedures spermatozoa require approximately $6 \mathrm{~h}$ to penetrate bovine oocytes in vitro. Although sequential fixations of oocytes following insemination were not performed in the present study, we predict that sperm capacitation and the acrosome reaction would require at least $4-6 \mathrm{~h}$ in our system. This leaves $2-4 \mathrm{~h}$ for the development of microtubule asters and sperm head decondensation before fixation of oocytes $8 \mathrm{~h}$ after insemination. Fertilized oocytes that had matured for $17 \mathrm{~h}$ predominately displayed sperm aster and chromatin decondensation patterns that were less developed. This could indicate either a delayed fertilization or a slower progression of events after fertilization. In contrast, oocytes that had matured for longer periods exhibited extensive microtubule asters and decondensed pronuclei following the same fertilization protocol. Similarly, experiments on delayed fertilization in mice by Fraser (1979) and Ishikawa et al. (1992) indicate a more rapid development to the pronuclear stage and accelerated embryonic development in mice undergoing delayed matings.

Further morphological evidence of oocyte variability due to ageing in maturation media comes from observing the cortical granule patterns. The distribution of cortical granules in bovine oocytes varied with the time of maturation, beginning with a clustered pattern which progressively became more uniformly distributed. No domain without cortical granules was observed associated with the metaphase II spindle, which is in contrast to patterns described by Ducibella et al. (1988) for mature mouse oocytes and Cherr et al. (1988) for hamster oocytes. Electron microscopy of mature mouse (Okada et al., 1986) and pig (Cran and Cheng, 1985) oocytes also indicate a cortical-granule-free domain. The clustered pattern of cortical granules in this study is similar to those reported by Kruip et al. (1983) using transmission electron microscopy of maturing bovine oocytes in vivo. These observations are also supported by Hyttel et al. (1988), who used transmission electron microscopy to observe clusters of cortical granules before fertilization in bovine oocytes matured in vitro for $28 \mathrm{~h}$. In our experiments, no bovine oocytes in any age group developed a cortical-granulefree area; this is comparable to cortical granule distribution patterns in domestic cats (Byers et al., 1992). Ooplasmic maturation of bovine oocytes and the ability to initiate sperm aster formation and sperm decondensation may be morphologically linked to progressive dispersal of cortical granules.

Cortical granules appear to be released at all stages of maturation in response to sperm penetration, although not to the same extent in some less mature oocytes. Therefore, a lack of cortical granule exocytosis does not explain the increase in polyspermic fertilization that occurred with increased maturation time. Rather, a decrease in the effectiveness of the cortical granule exudate must occur with increased maturation time.

Incomplete dispersal of exudate upon exocytosis during IVF has been reported in cattle and is associated with polyspermic fertilization of in vitro and in vivo matured oocytes (Hyttel et al., 1988, 1989). Cran and Cheng (1985) have observed similar patterns of cortical granule exudate following in vitro fertilization in pig oocytes. Failure to distribute these granules could lead to a defective formation of a cortical granule envelope, as described in mice by Dandekar and Talbot (1992), and thus to an ineffective block to polyspermy. Alternatively, the zona pellucida could become less responsive to the cortical granule contents as maturation time in vitro is increased. Zona hardening during ageing in vitro has been described in mice (Gianfortoni and Gulyas, 1985; Downs et al., 1986); although this phenomenon has not been described for bovine oocytes, it is possible that the ageing of the zona alters its response to the cortical granule exudate.

In summary, these experiments have demonstrated an effective fluorescent staining procedure for bovine oocyte cortical granules and its use in determining the cytoplasmic distribution during extended maturation periods. Cortical granules were progressively more distributed as the maturation time increased and were effectively released by sperm penetration, but were ineffective in preventing polyspermic fertilizations. Penetration of aged oocytes by spermatozoa results in the rapid development of a sperm aster and 
decondensed pronuclei, in contrast to undeveloped asters and pronuclei in less mature oocytes, suggesting an inability of less matured oocytes to respond to fertilization-induced intracellular signals. Both of these conditions may account for the subsequent lower rate of development of these zygotes to blastocysts. These results indicate that the time of incubation of spermatozoa with oocytes, sire selection and the age of oocytes at fertilization are all important factors in the success of in vitro fertilization of bovine oocytes. Manipulation of these parameters can reduce polyspermy, although this is most often accompanied by a decrease in total oocyte penetration rates and has little effect on monospermic fertilization.

The authors thank J. J. Balise for excellent technical assistance and Eastern A. I. Cooperative, Incorporated, Ithaca, NY for providing financial support and the frozen semen used in these experiments.

\section{References}

Bavister BD, Liebfried ML and Lieberman G (1983) Development of preimplantation embryos of the golden hamster in a defined culture medium Biology of Reproduction 28 235-247

Byers AP, Barone MA, Donoghue AM and Wildt DE (1992) Mature domestic cat oocyte does not express a cortical granule-free domain Biology of Reproduction 47 709-715

Cherr GN, Drobnis EZ and Katz DF (1988) Localization of cortical granule constituents before and after exocytosis in the hamster egg journal of Experimental Zoology 246 81-93

Chian RC, Nakahara H, Kiwa K and Funahashi H (1992) Fertilization and early cleavage in vitro of ageing bovine oocytes after maturation in culture Theriogenology $37665-672$

Cran DG and Cheng WTK (1985) Changes in cortical granules during porcine oocyte maturation Gamete Research $11311-319$

Cran DG and Esper CR (1990) Cortical granules and the cortical reaction in mammals Journal of Reproduction and Fertility Supplement 42 I77-188

Dandekar P and Talbot P (1992) Perivitelline space of mammalian oocytes: extracellular matrix of unfertilized oocytes and formation of a cortical granule envelope following fertilization Molecular Reproduction and Development 31 135-143

Dejarnette JM, Saacke RG, Bame J and Vogler CJ (1992) Accessory sperm: their importance to fertility and embryo quality, and attempts to alter their numbers in artificially inseminated cattle Journal of Animal Science 70 484-491

Dominko T and First NL (1992) Kinetics of bovine oocyte maturation allows selection for developmental competence and is affected by gonadotropins Theriogenology 37203

Downs SM, Schroeder AC and Eppig JJ (1986) Serum maintains the fertilizability of mouse oocytes matured in vitro by preventing hardening of the zona pellucida Gamete Research 15 I15-122

Ducibella T, Anderson E, Albertini DF, Aalberg J and Rangarajan S (1988) Quantitative studies of changes in cortical granule number and distribution in the mouse oocyte during meiotic maturation Developmental Biology 130 184-197

Fraser LR (1979) Rate of fertilization in vitro and subsequent nuclear development as a function of the post-ovulatory age of the mouse egg Journal of Reproduction and Fertility $55 \quad 153-160$

Gianfortoni JG and Gulyas BJ (1985) The effects of short term incubation (aging) of mouse on in vitro fertilization, zona solubility, and embryonic development Gamete Research 11 59-68

Hyttel P, Xu KP, Smith S and Greve T (1986) Ultrastructure of in-vitro oocyte maturation in cattle Journal of Reproduction and Fertility 78 615-625
Hyttel P, Xu KP and Greve T (1988) Ultrastructural abnormalities of in vitro fertilization of in vitro matured bovine oocyles Anatomy and Embryology 178 $47-52$

Hyttel P, Callesen H and Greve T (1989) A comparative ultrastructural study of in vivo versus in vitro fertilization of bovine oocytes Anatomy and Embryology $179435-442$

Ishikawa H, Katsuhiko $\mathrm{O}$ and Enda A (1992) Compensatory development in preimplantation mouse embryos derived from delayed mating Biology of Reproduction 47 782-784

Iwasaki S, Shioya Y, Masuda H, Hanada A and Nakahara T (1989) Incidence of chromosomal anomalies in early bovine embryos derived from in vitro fertilization Gamete Research 22 83-91

Kruip TAM, Cran DG, van Beneden TH and Dieleman SJ (1983) Structural changes in bovine oocytes during final maturation in vivo Gamete Research 8 29-47

Long CR, Pinto-Correia C, Duby RT, Ponce de Leon F, Boland MP, Roche JF and Robl JM (1993) Sperm aster formation and the first cell cycle in monospermic and polyspermic cow zygotes Molecular Reproduction and Development $36 \quad 23-32$

Nadir S, Saacke RG, Bame J, Mullins J and Degelos S (1993) Effect of freezing semen and dosage of sperm on number of accessory sperm, fertility and embryo quality in artificially inseminated cattle journal of Animal Science $7 \mathbf{1}$ 199-204

Ohgoda O, Niwa K, Yuhara M, Takahashi S and Kanoya K (1988) Variations in penetration rates in vitro of bovine follicular oocytes do not reflect conception rates after artificial insemination using frozen semen from different bulls Theriogenology 29 1375-1381

Okada A, Yanagimachi R and Yanagimachi H (1986) Development of a cortical granule-free area of cortex and the perivitelline space in the hamster oocyte during maturation and following ovulation Submicroscopic Cytology 18 233-247

Parrish JJ, Susko-Parrish JL, Leibfried-Rutledge ML, Critser ES, Eyestone WH and First WL (1986) Bovine in vitro fertilization with frozen-thawed semen Theriogenology 25 591-600

Parrish JJ, Susko-Parrish JL, Winer MA and First NL (1988) Capacitation of bovine sperm by heparin Biology of Reproduction 38 1171-1180

Pavlok A, Lucas-Hahn A and Niemann H (1992) Fertilization and developmental competence of bovine oocytes derived from different categories of antral follicles Molecular Reproduction and Development 31 63-67

Pinto-Correia C, Long C, Duby RT and Robl JM (1992) Development of polyspermic cow zygotes after multiple sperm aster formation Molecular Biology of the Cell 316 (Abstract)

Rosenkrans CF and First NL (1991) Culture of bovine zygotes to the blastocyst stage: effects of amino acids and vitamins Theriogenology 35266 (Abstract)

Saeki K, Kato H, Hosoi Y, Miyake M, Utsumi K and Iritani A (1991) Early morphological events of in vitro fertilized bovine oocytes with frozenthawed spermatozoa Theriogenology 35 1051-1058

Sathananthan AH and Trounson AO (1982) Ultrastructural observations on cortical granules in human follicular oocytes cultured in vitro Gamete Research 5 191-198

Schatten GC, Simerly C and Schatten H (1985) Microtubule configurations during fertilization, mitosis and early development in the mouse and the requirement for egg microtubule-mediated motility during mammalian fertilization Proceedings of the National Academy of Sciences USA $\mathbf{8 2}$ $4152-4156$

Susko-Parrish JL, Wheeler MB, Ax RL, First NL and Parrish JJ (1990) The effect of penicillamine, hypotaurine, epinephrine and sodium metabisulfite on bovine in vitro fertilization Theriogenology 33333

van der Westerlaken LAJ, de Wit AAC, van der Schans A, Eyestone WH and de Boer H (1992) Relationship between kinetics of polar body extrusion and developmental potential of bovine oocytes The 12th International Congress on Animal Reproduction. The Netherlands $1384-386$

Xu KP and Greve T (1988) A detailed analysis of early events during in-vitro fertilization of bovine follicular oocytes Journal of Reproduction and Fertility' 82 127-134 